\title{
FORMULACIÓN E IMPLEMENTACIÓN DE UN MODELO DE INNOVACIÓN EDUCATIVA PARA FORTALECER LAS CAPACIDADES EN ESTUDIANTES DE EDUCACIÓN MEDIA, EN LA RESOLUCIÓN DE PROBLEMAS CON EL USO DE TICS Y STEM
}

\author{
Formulation and implementation of an educational innovation model to strengthen middle \\ school student's abilities, in solving problems with the use of ICTs and STEM
}

\author{
Franck Sebastian Panche Carreño \\ fspanche@poligran.edu.co \\ Politécnico Grancolombiano \\ Matemáticas \\ Colombia
}

\section{Resumen}

El propósito de este estudio de casos es identificar, por medio de la observación, los documentos y registros, las competencias para la investigación y la resolución de problemas que desarrollaban estudiantes de educación media, al implementar un modelo de innovación educativa que evaluaba, estructuraba y refinaba las habilidades de pensamiento crítico, analítico y de trabajo en equipo, en escenarios donde las problemáticas locales (individuo - institución) eran el eje del proceso, bajo una estructura de pedagogía conceptual al usar las matemáticas y las TIC. El diseño de la investigación fue de tipo fenomenológico, se desarrolló en el Colegio Gimnasio el Lago de Bogotá con el acompañamiento de la Facultad de Ingeniería, Diseño e Innovación de la Institución Universitaria Politécnico Grancolombiano, con una muestra por conveniencia de 6 estudiantes de grado 10 y 11, durante el año académico de 2019. La técnica de análisis empleada fue la triangulación de los datos obtenidos con los instrumentos de medida, al implementar el modelo de innovación. El principal hallazgo del proyecto fue la formulación de una propuesta pedagógica y didáctica innovadora en la que el conocimiento de las ciencias, la tecnología, la ingeniería y las matemáticas permitieron fomentar y fortalecer el desarrollo de competencias para la investigación y la resolución de problemas, lo cual contribuye fuertemente al desarrollo del país y su sostenibilidad responsable, además de constituirse en un referente como modelo de innovación educativa para la educación media. Dentro de los resultados obtenidos que impactan al estudiante, se encuentran la participación en encuentros juveniles matemáticos a nivel de poster y de proyección como formulador de soluciones a problemas de la vida cotidiana de la institución.

Palabras clave:

Innovación educativa, STEM, competencias para la investigación y resolución de problemas. 


\section{INTRODUCCIÓN}

La formulación e implementación de metodologías educativas estratégicas para aproximar al estudiante a la interpretación consciente y responsable de su entorno, involucrándolo como agente generador de cambios a través de la formulación de modelos con el uso de herramientas tecnológicas, las ciencias, la ingeniería y las matemáticas para no solo visualizar las problemáticas directas de su realidad, sino proponer soluciones óptimas de seguimiento y resolución, implican estructurar procesos que converjan en innovaciones educativas (Arteaga, Armada \& Del Sol, 2016), entendidas como el conjunto o colección de ideas, procesos y estrategias con inmersión sistémica hacia la introducción de cambios educativos tangibles y visibles para los participes del proceso enseñanza aprendizaje (De León, 2005, p. 11), que permeen, sensibilicen y promuevan en los estudiantes el deseo por aprender en prospectiva al mundo que éstos afrontarán en sus futuros.

Ante la cuarta revolución industrial, se está evidenciando e identificando que las carreras del futuro demandarán a los individuos un dominio en el manejo de información para predecir e inferir resultados a partir de modelos y desarrollos en programación con elementos de inteligencia artificial, para lograr el desarrollo de los países y su sostenibilidad responsable desde lo social, económico, político y ambiental, en beneficio de la sociedad (Semana, 2019). Los obstáculos para generar ambientes investigativos en la educación se radican en La imprecisión, diversidad y divergencia de las concepciones sobre la enseñanza para la investigación escolar que suelen expresar los maestros, que les conduce a implementar estrategias de consulta que no facilitan la indagación y estructuran la enseñanza bajo esa misma etiqueta De León, P. C., González, G. T., \& Estrada, F. J. P. (2011).

La investigación escolar a es un proceso de reflexión por el cual se concibe una problema, una necesidad, donde se desea mejorar la práctica o la comprensión personal de dicha problemática, el estudiante en ejercicio lleva a cabo un estudio -en esencia, para conceptualizar con claridad el eje del problema; en segundo lugar, para especificar un plan de acción [...] Dadas las condiciones se emplean lenguajes generales científicos o axiomático(Alzate \& Góez, 2017; Campillo \& Martínez, 2017; Oviedo \& Silva, 2017; Rojas, Rodríguez, Giraldoth, Chacín, \& Reyes, 2017; Ruiloba, 2014). Luego se formula una evaluación continua para comprobar y establecer la efectividad de la acción tomada. Bajo esta idea, por último, los participantes reflexionan, explican los progresos y comunican estos resultados a la comunidad de investigadores en formación escolar de la acción o problema. La investigación escolar es un estudio científico auto reflexivo de los estudiante para mejorar sus practicas académicas (McKernan, 1999, p. 25), este entorno nos evidencia un escenario donde la problematica es el eje, un acercamiento a competencias investigativas y consecuentemente a la resolución de problemas.

Por otra parte, D’Antoni (2010) justifica que la interdisciplina o el conocimiento transversal facilita los canales afectivos del educando con las necesidades de su mundo inmediato, en donde las competencias en investigación y relsoución de problemas son un constructo de las herramientas docente, sin embargo, la rigurosidad de los procesos se imposibilitan por el vacio conceptual en el manejo de información, por ello, se hace imperante formar estrategias para el manejo de esta. Es decir, el desarrollo de competencias en investigación y resolución de problemas se edifica en fuertes bases cognitivas y en el análisis y recolección de información, componentes poco explorados en la vida escolar.

Debido a esto, el presente estudio buscaba dar respuesta a la pregunta ¿cuáles son las competencias para la investigación y la resolución de problemas que puede desarrollar un estudiante de educación media al implementar un modelo de innovación educativa enmarcado en el uso de las ciencias, la tecnología, la ingeniería, las matemáticas y las TIC?, lo cual permitirá a diversas instituciones de educación media establecer estrategias 
para incorporar el modelo y desarrollar competencias que fomenten el desarrollo del pensamiento crítico, creativo y analítico que son fuentes para formar generaciones conscientes y comprometidas con su entorno personal y social.

\section{Método}

El proyecto se enmarca en una investigación con un enfoque cualitativo por identificar y caracterizar las competencias que pueden desarrollar los estudiantes de educación media de un colegio, para investigar y resolver problemas del entorno, que estén a su alcance, haciendo uso de las TIC, las ciencias, la ingeniería y las matemáticas, a través de la implementación de un modelo de innovación educativa. El diseño es de tipo fenomenológico por pretender identificar características y regularidades a partir de lo que los estudiantes experimenten al estar inmersos en el modelo de innovación educativa enmarcado en el uso de las ciencias, la tecnología, la ingeniería, las matemáticas y las TIC (Carlos Eduardo Daza-Orozco, 2019).

La investigación se desarrolla en el Colegio Gimnasio el Lago, con el acompañamiento de la Facultad de Ingeniería, Diseño e Innovación de la Institución Universitaria Politécnico Grancolombiano (Bogotá), con estudiantes de los grados décimo y once. La muestra se estructuró por conveniencia y está conformada por seis estudiantes junto con el docente, que a la vez cumple el rol de investigador(Daza-Orozco, C; Cera-Ochoa, 2018; C E Daza-Orozco, 2015; Daza-Orozco E.; Ramírez-Varela, F.; Arboleda-Muñoz, G.; Gil-Ángel, G.; Villada-Castillo, H.; Portela-Guarín, H.; García-Fernández, J.; Estévez-Ceballos, J.; Infante- Castillo, A.; Rodríguez-Cuberos, E.; Cascante-Gatgens, A.; Barrien, M., 2019). La técnica de análisis de datos a usar fue la triangulación de datos, en ella se registraron los constructos del estudio, las regularidades y las características, para luego establecer las competencias que podían desarrollar con la implementación del modelo(Luis Martín; Trujillo-Flórez et al., 2019).

La propuesta pedagógica y didáctica se fundamentó en el modelo que expone el proyecto educativo institucional del colegio en el marco de la pedagogía conceptual, que consiste en la caracterización de los instrumentos de conocimiento y las operaciones intelectuales propias de cada momento del desarrollo humano; a través de lo cognitivo aplicantando estructuras mentefactuales que llevan a el estusiante a la visualización de problematícas y representarlas en la generalización del metalenguaje y aplicación de modelos matemáticos que difieren de los estandares escolares y fomentan espacios autodidáctas que le permiten exclareser, relievar y estructurar hipotesis que aborda el pensamiento analítico, en referencia a la formulación y argumentación de modelos matemáticos con el propósito de entender las variables, métodos y herramientas matemáticas y tecnológicas para entender los tópicos problematizadores; el afectivo, puesto que dichas problemáticas -inmediatas y de relevancia a las necesidades sociales del esudiante- generan disonancia cognotiva que le conllevan a deconstruir su posición frente en apoyo con el trabajo en equipo, puesto que le obliga a a ampliar su espectro de acción a través de las vivencia de sus pares en contextos similares con dinamicas exogenas y el expresivo dado que justificar su posición y las posibles repercusiones de su análisis y vivencia constituye en el estudiante una perspectiva crítica de las consecuencias de la problemática y soluciones ideales(Luis Martín; Trujillo-Flórez et al., 2018).

Las problemáticas en las que intervienen los estudiantes son canónicas y universales dentro de su contexto, por ende, necesutan de un lenguaje propicio que concatene el traiángulo de la mente humana (De Zubiría, 2006) de allí se justifica el desrrollo constnte de las STEM (ciencia, Tecnología, Ingenieria y Matemáticas) puesto que permite utilizar un lenguaje universal libre de ambigüedad, de esto se propone el siguiente esquema(E. NormanAcevedo, 2018). 


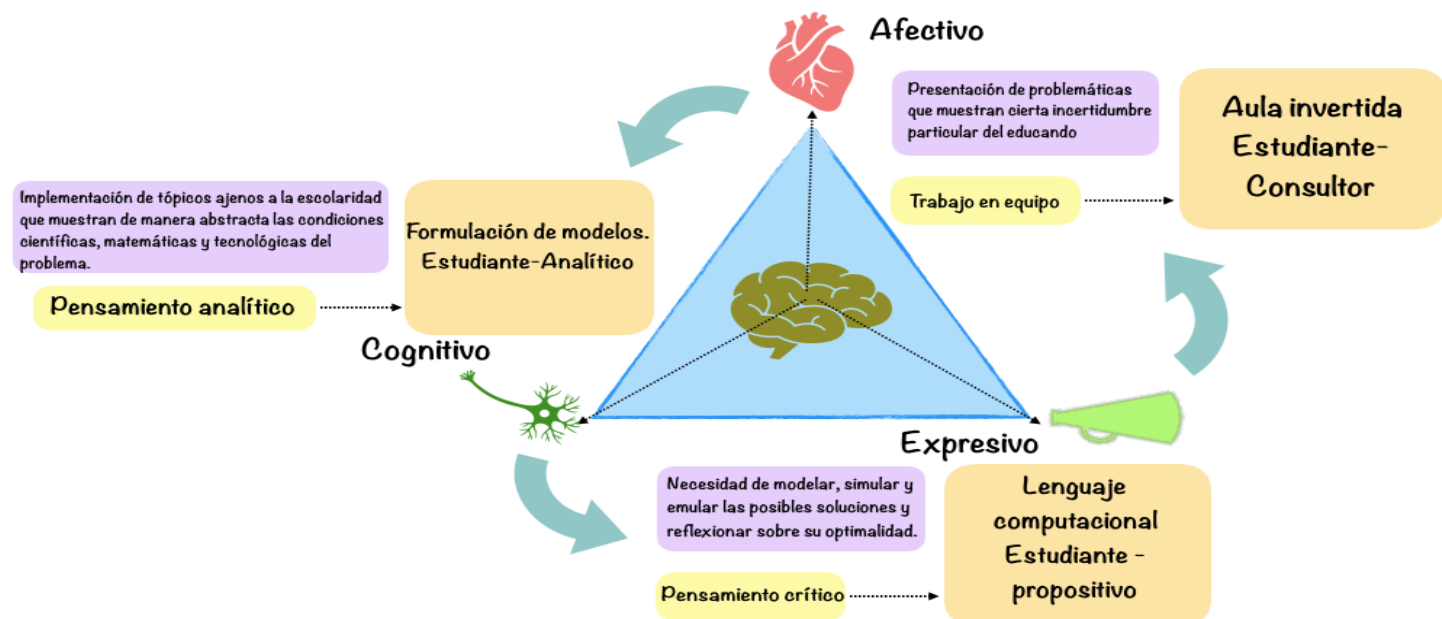

Diagrama 1 Propuesta de adaptación del modelo pedagógico adaptado al desarrollo de competencias con el uso de la ciencia, tecnología, ingeniería y matemáticas.

Dada la inmersión de la problemática dentro de la cotidianidad del quehacer académico del estudiante se evidenció, que en base a las habilidades del pensamiento anlítico, crítico y el trabajo en equipo proliferan algunas competencias própias para la investigación y la resolución de problemas, como muestra el siguiente cuadro:

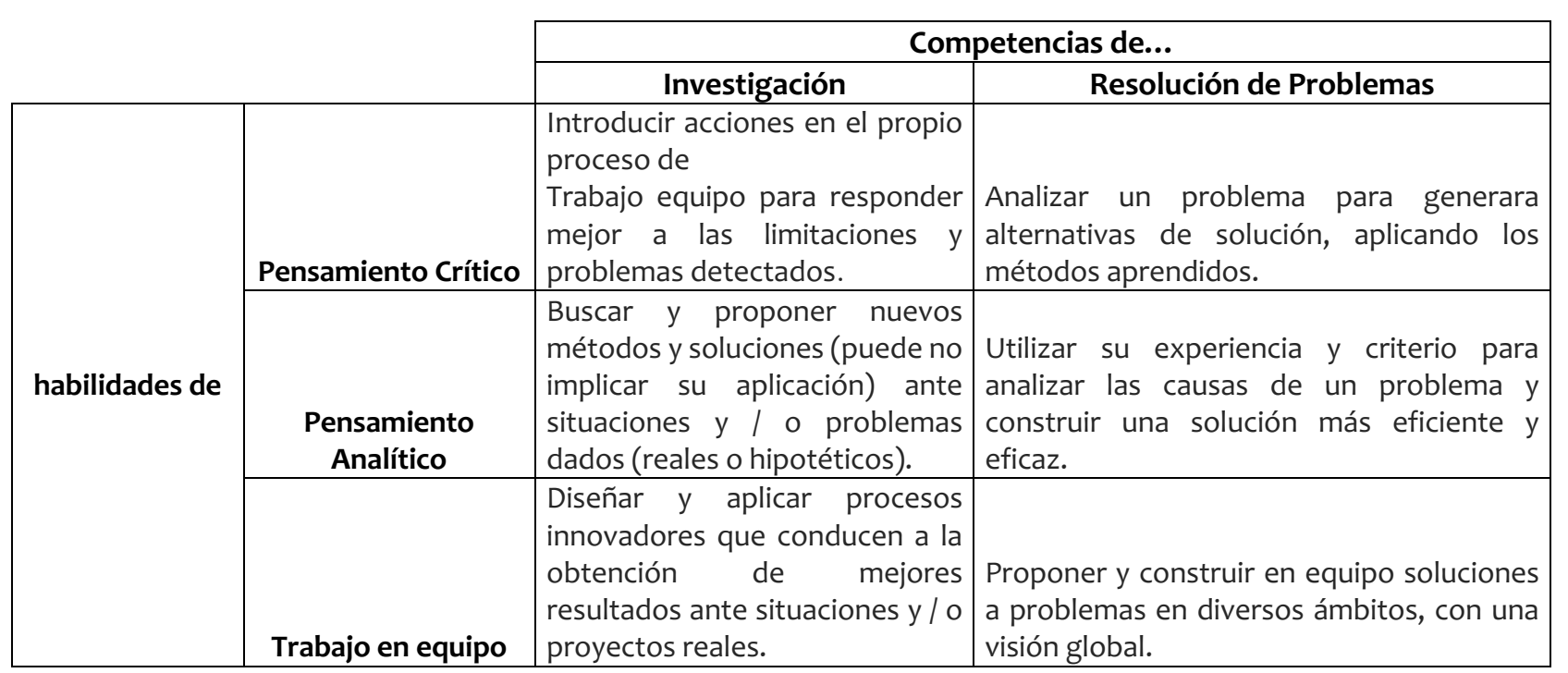

Cuadro 2 Relación de las competencias en investigación y resolución de problemas mediante la cualificación del pensamiento crítico, analítico y el trabajo en equipo.

\section{Resultados}

La inmersión del estudiantado en el marco de problemáticas inmediatas y la estructuración metacognitiva a través de la pedagogía conceptual, en el entorno de herramientas como: Tic y las ciencias, la tecnología, la ingeniería, las matemáticas (STEM), lleva a la resolución de problemas, y la modelación de dichas soluciones, esto permite no solo al estudiante interactuar en su contexto como un agente generador de cambios sino a su vez, transformar a los actores del proceso enseñanza aprendizaje, permitiendo generar ambientes propicios para la interdisciplinariedad y el trabajo en equipo; por lo anterior se esquematiza la ruta de observación y sus directos responsables, como se muestra diagrama 2.

Los resultados preliminares del estudio corresponden a:1) una propuesta pedagógica y didáctica innovadora para la iniciación en procesos de investigación y el fortalecimiento de habilidades cognitivas, tecnológicas, de 
colaboración y comunicación en estudiantes de educación media a través de la resolución de problemáticas del entorno en las que el conocimiento de las ciencias, la tecnología, la ingeniería, las matemáticas (STEM) y el uso de TIC se constituyan como herramientas que posibilitan el planteamiento de una solución, 2) la participación de una de las estudiantes que ha estado inmersa en el modelo de innovación educativa en el octavo encuentro de matemáticas juveniles (2019), en el cual se hizo una socialización del trabajo y los resultados obtenidos en el estudio Grafo reticular: una vista del algoritmo de Djikstra y la triangulación de caminos, y 3) la construcción del modelo de innovación educativa, el cual contempla la definición de los diferentes elementos del modelo, el plan operativo y la ruta de acción.

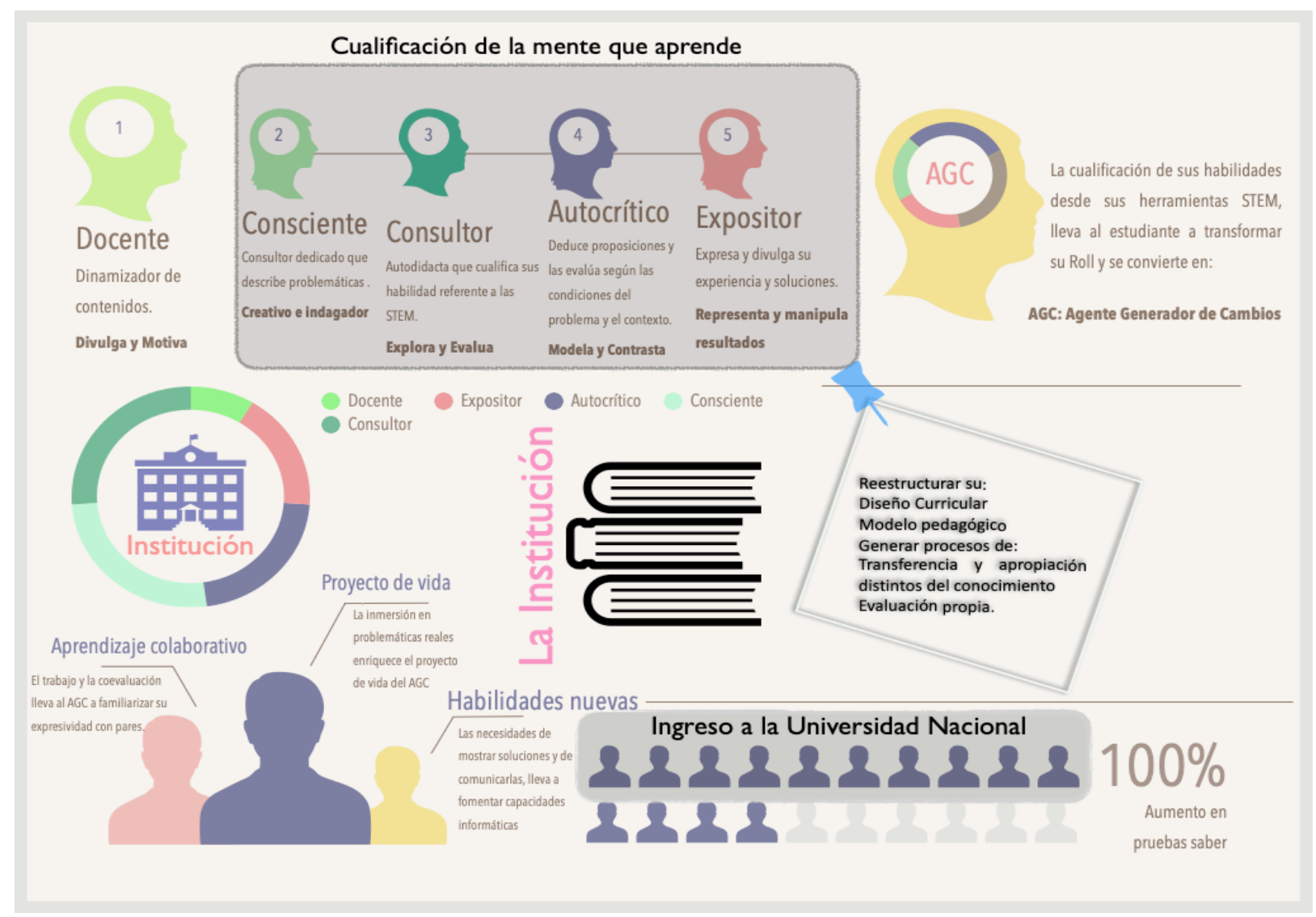

Diagrama 2 Esquema de resultados según la cualificación de los estudiantes en habilidades STEM y las repercusiones institucionales y grupales.

El modelo de innovación educativa permitió establecer entornos de aprendizaje que faciliten los canales de divulgación del estudiantado participe, estos mecanismos se implementaron desde dos perspectivas: La exploración de talentos científicos y la exposición de las técnicas trabajadas a la comunidad educativa, por ello, se hizo necesario, constituir un equipo institucional denotado club de indagación, y exploración de talentos matemáticos Sophie de Germain el cual fue el medio para trascender las ideas de los estudiante, a su vez, se constituyo el concurso intercolegiados de demostración de identidades trigonométricas David Hilbert, con el que se permite compartir con las demás instituciones del sector (Díaz, 2016; Mendoza, 2016; Moreno, Cediel, \& Herrera, 2016; Eduardo Norman-Acevedo, 2019; Obando, 2015; Peña-García, 2019).

Discusión y Conclusión 
La STEM (Matemáticas, Ciencias, tecnología e Ingeniería) permite al educando desarrollar habilidades que le abren un camino dirigido a las necesidades del mundo de hoy, no solo en el campo académico e industrial, sino a su vez en el campo social como agente de cambio real, su cosmovisión y la toma de decisiones, por ello es imperante la transformación de los roles en todos los agentes participes del proceso enseñanza aprendizaje, focalizando al docente no como expositor de contenidos, sino como divulgador experto de problemáticas cercanas, por ende, las preguntas generadoras facilitan dicho camino.

Las mentes que transforman nuestro mundo son aquellas que divisan horizonte macro y micro espaciales, es decir, son mentes que relacionan contextos reales en ideas abstractas propias de evolucionar, por lo mismo, el trabajo en equipo. Las ideas creativas requieren del desarrollo y cualificación de competencias en el pensamiento crítico y analítico, siendo estos los insumos de los agentes generadores de cambio.

La innovación educativa no requiere de enormes recursos materiales, requiere mentes idóneas con perspectivas diferentes que sean mediadores intergeneracionales y propensas a la mundialización con fuertes conocimientos en manejo de información como demanda la sociedad contemporánea, siendo las STEM un lenguaje ideal para la comunicación de estas mentes. Por la naturaleza del saber natural de la muestra de estudiantes solo se enfatizo en las matemáticas y la tecnología, sin embargo, es imperante fortalecer e incorporar herramientas científicas teóricas y aplicadas como es la ingeniería

Por otra parte, para tomar decisiones referentes al alcance de las repercusiones del modelo de innovación educativa es demandante diseñar instrumentos de medición que permitan realizar un seguimiento idóneo del modelo y así llevar a la independencia y/o idempotencia respecto al enfoque pedagógico y estandarizar modelo de innovación educativa sobre bases socio-científicas.

\section{Referencias bibliográficas}

Alzate, D. M. O., \& Góez, Á. M. B. (2017). LA NUEVA MISIÓN DE LA UNIVERSIDAD. CONTEXTUALIZACIÓN Y RESULTADOS: CASOS DE TRES UNIVERSIDADES PÚBLICAS COLOMBIANASLA NUEVA MISIÓN DE LA UNIVERSIDAD. CONTEXTUALIZACIÓN Y RESULTADOS: CASOS DE TRES UNIVERSIDADES PÚBLICAS COLOMBIANAS-The new mission of... Panorama, 11(20), 82-94. https://doi.org/10.15765/pnrm.v11i20.1010

Arteaga, E., Armada, L., \& Del Sol, J. (2016). Teaching science in the new millennium. Challenges and suggestions. Revista Universidad y Sociedad, 8(1), 169-176. Recuperado en 02 de octubre de 2019, de http://scielo.sld.cu/scielo.php?script=sci_arttext\&pid=S2218-36202016000100025\&lng=es\&tIng=en

Bartolotta, S. (2017). Cuarta revolución industrial y educación en el tercer milenio: retos para una escuela de calidad. Recuperado de https://www.oei.es/historico/divulgacioncientifica/?Cuarta-revolucion-industrialy-educacion-en-el-tercer-milenio-retos-para-una

Campillo, É. T. G., \& Martínez, M. de J. G. (2017). METODOLOGÍA DOCENTE: INCIDENCIA EN LA APATÍA DE LOS ESTUDIANTES HACIA LAS CIENCIAS SOCIALES - Educational methodology: incidence in students apathy towards social sciences. Panorama, 11(21), 16-25. https://doi.org/10.15765/pnrm.v11i21.1049

D’Antoni, M., \& Arguedas, J. F. S. (2010). Experiencias de investigación en la secundaria: una visión desde adentro. Temas de Nuestra América. Revista de Estudios Latinoamericanos, 26(49), 71-86.

Daza-Orozco, CE. (2019). Iniciación científica: conceptualización, metodologías y buenas prácticas. Bogotá. Politécnico Grancolombiano. https://doi.org/10.13140/RG.2.2.20812.23684

Daza-Orozco, CE. (2015). La Investigación en los tiempos del acceso abierto. Silogismo. Retrieved from http://www.cide.edu.co/ojs/index.php/silogismo/article/view/294

Daza-Orozco, CE; Cera-Ochoa, RA. (2018). Escritura con estilo: Guía práctica para publicar científicamente. Bogotá: Editorial San Mateo. Retrieved from http://palma.sanmateo.edu.co/index.php/catalogo/series/41-escritura-con-estilo-guia-practica-parapublicar-cientificamente 
Daza-Orozco, Carlos Eduardo. (2019). INICIACIÓN CIENTíFICA Conceptualización, metodologías y buenas prácticas (1st ed.; S. C. de I. e I. F. (SCOIF), Ed.). Retrieved from http://www.scoif.com/iniciacion-cientifica/ De León, P. C. (2005). La innovación educativa (Vol. 4). Madrid: Ediciones AKAL.

De León, P. C., González, G. T., \& Estrada, F. J. P. (2011). Análisis de obstáculos y dificultades de profesores y estudiantes en la utilización de enfoques de investigación escolar. Investigación en la Escuela, (73), 5-26.

De Zubiría Samper, J. (2006). Los modelos pedagógicos: hacia una pedagogía dialogante. Coop. Editorial Magisterio.

Díaz, A. M. (2016). Evaluación de la propuesta de enseñanza de las áreas de lenguaje y matemáticas en la Institución Educativa San José del Pantano. Panorama, 9(17), 25-39. https://doi.org/10.15765/pnrm.vgi17.789

Hammersley,J.M.(1968). On the enfeeblement of mathematical skills by modern mathematics and by similar soft intellectual trash in schools and universities. Educational Studies in Mathematics, 1(1-2), 17-17.

McKernan, J. (1999). Investigación-acción y curriculum. Madrid: Morata.

Mendoza, J. A. G. (2016). Empresa privada: principal socio en el posconflicto y la construcción de la paz. Panorama, 10(18), 84-92. https://doi.org/10.15765/pnrm.v10i18.823

Moreno, K. G., Cediel, M. C. F., \& Herrera, L. Y. V. (2016). Emociones en niños y adolescentes desde la experiencia del desplazamiento y la vinculación a los grupos armados en Colombia. Panorama, 10(19), 85-96. https://doi.org/10.15765/pnrm.v10i19.834

Norman-Acevedo, E. (2018). Rompiendo Barreras, 10 Años de la Educación Virtual en el Politécnico Grancolombiano. (primera; Eduardo Norman-Acevedo, Ed.). Retrieved from http://alejandria.poligran.edu.co/handle/10823/1146

Norman-Acevedo, Eduardo. (2019). NUEVOS LENGUAJES PARA APRENDIZAJE VIRTUAL HERRAMIENTAS PARA LOS ESCENARIOS DE APRENDIZAJE. Panorama, 13(24), 5-7. https://doi.org/10.15765/pnrm.v13i24.1214

Obando, C. R. C. (2015). Movimientos barriales: reivindicación del derecho a la ciudad. Panorama, 9(16), 66-76. https://doi.org/10.15765/pnrm.vgi16.640

Oviedo, L. B., \& Silva, M. C. (2017). LA INVESTIGACIÓN ACCIÓN Y EL APRENDIZAJE POR PROYECTOS EN EL MARCO DEL MODELO PEDAGÓGICO ENSEÑANZA PARA LA COMPRENSIÓN. EXPERIENCIA DEL COLEGIO VISIÓN MUNDIAL EN COMUNIDADES VULNERABLES DE MONTERÍA-Action research and Project Based Learning in ... Panorama, 11(21), 38-51. https://doi.org/10.15765/pnrm.v11i21.1053

Peña-García, S. N. (2019). EL DESAFÍO DE LA COMPRENSIÓN LECTORA EN LA EDUCACIÓN PRIMARIA - The challenge of reading comprehension in primary education. Panorama, 13(24), 42-56. https://doi.org/10.15765/pnrm.v13i24.1205

Rojas, M. E. V. de, Rodríguez, H. J., Giraldoth, D. del V., Chacín, O. J. R., \& Reyes, D. D. J. (2017). GESTIÓN DEL DIRECTOR PARA EL FORTALECIMIENTO DEL PROCESO EDUCATIVO EN LAS ESCUELAS PRIMARIAS Director's management for the educational strengthening of primary schools. Panorama, 11(20), 7-18. https://doi.org/10.15765/pnrm.v11i20.976

Ruiloba, J. M. (2014). Sistema político local en tiempos difíciles: el caso español. Panorama, 8(14), 98-108. https://doi.org/10.15765/pnrm.v8i14.501

Semana (25 de enero de 2019). ¿Qué es la cuarta revolución industrial y por qué va a cambiar a la educación? Semana, (39).

Stillman, G. A., Kaiser, G., Blum, W., \& Brown, J. P. (Eds.). (2013). Teaching mathematical modelling: Connecting to research and practice. Springer Science \& Business Media.

Trujillo-Flórez, Luis Martín;, Bernal Yermanos, M. Á., Escobar Castro, J. A., González Triana, M. C., Gutiérrez Carvajal, J. P., Gutiérrez Vanegas, J. C., ... Torres López, A. L. (2019). Experiencias de innovación educativa - Tomo 3 (1st ed.; Luis Martín Trujillo-Flórez, Ed.). Bogotá: Politécnico Grancolombiano.

Trujillo-Flórez, Luis Martín;, Martínez Contreras, R. M., Espitia López, H., Rojas Paredes, J. C., Vargas Leguizamón, Y. R., \& Castro Cabal, G. A. (2018). Experiencias de innovación educativa (1st ed.; L. M. Flórez-Trujillo, Ed.). Bogotá: Politécnico Grancolombiano.

UNESDOC. (2016). Educación 2030: Declaración de Incheon y Marco de Acción para la realización del Objetivo de Desarrollo Sostenible 4: Garantizar una educación inclusiva y equitativa de calidad y promover 
oportunidades de aprendizaje para todos. Recuperado de https://unesdoc.unesco.org/ark:/48223/pfooo0245656_spa

Villa, A. P., \& Poblete, M. M.(2007). Aprendizaje basado en competencias. Una propuesta para la evaluación de las competencias genéricas. 